\title{
STRUCTURAL SPECIFICITIES FOR THE ACTIVE TRANSPORT SYSTEM OF THIAMINE IN RAT SMALL INTESTINE
}

\author{
Toru Komal and Hideyo SHINDo ${ }^{1}$ \\ Central Research Laboratories, Sankyo Co., Ltd., Shinagawa-ku, Tokyo 140
}

(Received April 18, 1974)

\begin{abstract}
The structural specificity of the thiamine transport system in the rat small intestine was examined by studying the ability of thiamine analogues to inhibit the unidirectional influx of ${ }^{35} \mathrm{~S}$-thiamine from medium to everted ring segments of the rat small intestine.

The results indicated that the amino group and the quarternary nitrogen atom are both required for the transport of thiamine by the rat small intestine, and that 2'-methyl and 5-hydroxyethyl groups significantly influence the binding of thiamine molecule to the carrier. The C-2 position of thiazole moiety, which is an active site of coenzyme reaction of thiamine pyrophosphate, seems to play no role in thiamine transport. The thiamine transport system was found to be different from the sugar and amino acid transport systems.
\end{abstract}

A conceptual model of the thiamine binding to the carrier was presented.

It has been known that the affinity of sugars (1) or amino acids (2) to the membrane carriers which mediate the active transport has a high specificity with respect to the chemical structure of substrates.

As described in the preceding paper (3), thiamine was proved to be actively transported by a specific carrier from the rat small intestine. It was shown that thiamine analogues such as pyrithiamine and chloroethylthiamine competitively inhibited the thiamine active transport, while oxythiamine did not affect the transport, indicating that a specific structure of thiamine molecule is involved in the interaction of thiamine with the binding site of the carrier.

In this paper, the inhibitory effect of various thiamine-related compounds on the thiamine active transport by the rat small intestine was examined, in order to clarify the structural requirement for the active transport of thiamine.

1 駒井 亨, 進藤英世 
In addition, a relationship between the thiamine transport system and the transport systems of sugars and amino acids was examined.

\section{EXPERIMENTAL}

Materials. ${ }^{35} \mathrm{~S}$-Thiamine was purchased from Radiochemical Center, Amersham, England. The specific activity was 300 to $470 \mu \mathrm{Ci} / \mathrm{mg}$. Thiamine monophosphate, thiamine pyrophosphate, pyrithiamine and oxythiamine were the commercial products. Other thiamine analogues used were prepared in our laboratory.

Experiments on thiamine uptake by everted intestinal ring segments. Male Wistar-Imamichi rats weighing 200 to $250 \mathrm{~g}$ were used after $16 \mathrm{hr}$ fasting. The tissue accumulation of ${ }^{35} \mathrm{~S}$-thiamine was examined as previously described (3). The initial concentration of ${ }^{35} \mathrm{~S}$-thiamine in the medium was about $0.6 \times 10^{-6} \mathrm{M}$ and that of thiamine-related compounds was $6 \times 10^{-6} \mathrm{M}$. The reaction vessels were incubated at $37^{\circ} \mathrm{C}$ for $30 \mathrm{~min}$ after preincubation for $10 \mathrm{~min}$ under $95 \%$ $\mathrm{O}_{2}-5 \% \mathrm{CO}_{2}$ atmosphere.

When the effects of sugars of amino acids were examined, Krebs-Henseleit bicarbonate buffer was used without adding glucose, and the concentration of sugars or amino acids was $10 \mathrm{~mm}$.

When the effect of $\mathrm{pH}$ values of the medium on thiamine transport was examined, Krebs-Henseleit bicarbonate buffer lacking $\mathrm{CaCl}_{2}$ was used after adjusting the $\mathrm{pH}$ value by adding $1 \mathrm{~N} \mathrm{HCl}$ or $1 \mathrm{~N} \mathrm{NaOH}$.

Paper electrophoresis of thiamine. Paper electrophoresis of ${ }^{35}$-thiamine was performed with the type IIC paper electrophoresis apparatus of Toyo Roshi Co., using Toyo filter paper No. 51A at 13 volts $/ \mathrm{cm}$ for $3 \mathrm{hr}$. Picric acid and caffein were used as the reference compounds. The former has a single negative charge over the $\mathrm{pH}$ range studied and was employed to correct for the electrophoretic mobility. The latter has no electrical charge over the $\mathrm{pH}$ range tested and was used to correct for electro-osmotic movement of the solvent. Thus, the mobility of thiamine is expressed as the ratio of the distance between thiamine and caffein to that between picric acid and caffein. ${ }^{35} \mathrm{~S}$-Thiamine was detected by the autoradiography.

\section{RESULTS}

Inhibition of thiamine transport by various thiamine-related compounds

The inhibitory effect of various thiamine-related compounds on the thiamine active transport by the rat small intestine was examined and the results were listed in Table 1.

a) Effect of amino, group. As shown in Table 1-a, thiamine and chloroethylthiamine inhibit the transport of ${ }^{35}$ S-thiamine by 80.73 and $54.26 \%$, respectively. However, replacement of the amino group of pyrimidine moiety by 
Table 1. Effect of thiamine-related compounds on ${ }^{35} \mathrm{~S}$-thiamine uptake by rat small intestine. ${ }^{\mathrm{a}}$

\begin{tabular}{|c|c|c|c|}
\hline & Thiamine analogue & $\begin{array}{l}\text { Inhibition } \\
(\% \pm \text { S.E. })\end{array}$ & $\mathrm{n}^{\mathrm{b}}$ \\
\hline \multirow[t]{5}{*}{ a) } & Modification of amino group & & \\
\hline & thiamine & $80.73 \pm 0.99$ & 12 \\
\hline & chloroethylthiamine & $54.26 \pm 2.49$ & 12 \\
\hline & oxythiamine & $12.82 \pm 3.44$ & 8 \\
\hline & oxychloroethylthiamine & $5.28 \pm 3.89$ & 4 \\
\hline \multirow[t]{4}{*}{ b) } & Modification of $2^{\prime}$-methyl group & & \\
\hline & chloroethylthiamine & $54.26 \pm 2.49$ & 12 \\
\hline & 2'-ethyl-chloroethylthiamine & $48.20 \pm 3.80$ & 4 \\
\hline & 2'-propyl-chloroethylthiamine & $26.67 \pm 1.10$ & 4 \\
\hline \multirow[t]{4}{*}{ c) } & Modification of quarternary nitrogen & & \\
\hline & thiamine anhydride & $0.08 \pm 3.33$ & 4 \\
\hline & dihydrothiamine & $0.00 \pm 4.76$ & 4 \\
\hline & thiamine disulfide & $2.10 \pm 2.12$ & 4 \\
\hline \multirow[t]{3}{*}{ d) } & Modification at 2-position of thiazole moiety & & \\
\hline & 2-hydroxyethylthiamine & $78.42 \pm 2.03$ & 4 \\
\hline & 2-methylthiamine & $78.18 \pm 1.93$ & 4 \\
\hline \multirow[t]{9}{*}{ e) } & Modification of 5-position of thiazole moiety & & \\
\hline & thiamine & $80.73 \pm 0.99$ & 12 \\
\hline & thiamine monophosphate & $73.39 \pm 2.78$ & 4 \\
\hline & thiamine pyrophosphate & $75.07 \pm 3.13$ & 4 \\
\hline & dehydroxyethylthiamine & $64.00 \pm 1.16$ & 4 \\
\hline & 5-methylthiamine & $67.58 \pm 1.69$ & 4 \\
\hline & 5-hydroxymethylthiamine & $58.05 \pm 3.78$ & 4 \\
\hline & chloroethylthiamine & $54.26 \pm 2.49$ & 12 \\
\hline & O-benzylthiamine & $43.91 \pm 1.50$ & 4 \\
\hline \multirow[t]{5}{*}{ g) } & Effect of pyrimidine and thiazole moieties & & \\
\hline & 2-methyl-4-amino-5-aminomethyl pyrimidine (Pym.) & $2.73 \pm 4.51$ & 4 \\
\hline & 4-methyl-5-hydroxyethyl thiazole & $-1.20 \pm 2.31$ & 4 \\
\hline & N,4-dimethyl-5-hydroxyethyl thiazole (Thyz.) & $-2.80 \pm 1.76$ & 4 \\
\hline & Pym. + Thyz. & $3.16 \pm 1.74$ & 4 \\
\hline
\end{tabular}

${ }^{a}$ Everted ring segments of the rat small intestine were incubated in $15 \mathrm{ml}$ KrebsHenseleit bicarbonate buffer containing ${ }^{35} \mathrm{~S}$-thiamine $\left(0.6 \times 10^{-6} \mathrm{M}\right)$ in the presence or absence of thiamine-related compounds $\left(6 \times 10^{-6} \mathrm{M}\right)$ for $30 \mathrm{~min}$ at $37^{\circ} \mathrm{C}$ under $95 \% \mathrm{O}_{2^{-}}$ $5 \% \mathrm{CO}_{2}$ atmosphere. The structure of thiamine is numbered as follows.

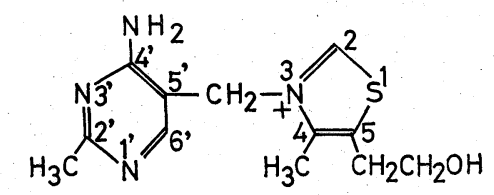

b Number of experiments. 
hydroxy group significantly reduced their inhibitory effects. This result indicates that the amino group plays an important role in the binding of thiamine molecule to the carrier.

b) Effect of 2'-methyl group. In order to examine the effect of $2^{\prime}$-methyl group, the inhibition of thiamine transport by $2^{\prime}$-methyl-, $2^{\prime}$-ethyl- and $2^{\prime}$-propylchloroethylthiamine was compared. As shown in Table 1-b, the inhibitory effect was decreased in the order of increasing the bulk of the group at the 2'position, suggesting a steric hindrance of the substituent. It seems likely, therefore, that 2 '-methyl group of thiamine is enveloped in the carrier molecule.

c) Effect of quarternary nitrogen of thiazole moiety. Thiamine has a quarternary nitrogen atom in the thiazole moiety and its formal charge at physiological $\mathrm{pH}$ range is +1 . In order to elucidate the effect of quarternary nitrogen, the inhibitory effect on thiamine transport was examined by thiamine anhydride, dihydrothiamine and thiamine disulfide, all of which lack the quarternary nitrogen atom. As shown in Table 1-c, these analogues did not inhibit thiamine uptake to any appreciable extent, indicating that the quarternary nitrogen is essential for the thiamine transport.

d) Effect of 2-hydrogen in thiazole moiety. As listed in Table 1-d, the thiamine analogues substituted at 2-position by a bulky group such as methyl or hydroxyethyl groups inhibited thiamine transport in the similar extent to that of the corresponding analogue having hydrogen atom at this position (thiamine). This result indicates that the substitution by a bulky group at the 2-position does not influence the affinity of thiamine molecule to the binding site of the carrier and suggests that the 2-position of thiazole moiety is not involved in the interaction with the carrier.

e) Effect of the group at the 5-position of thiazole moiety. All the thiamine analogues with varing group at the 5 -position of thiazole moiety inhibited ${ }^{35} \mathrm{~S}$ thiamine transport appreciably. Thiamine was the most potent inhibitor among them and thiamine pyrophosphate and thiamine monophosphate showed a strong inhibition of more than $70 \%$ (Table 1-e). On the other hand, replacement of the hydroxyethyl group of thiamine by smaller groups as hydrogen, hydroxymethyl and methyl groups reduced the inhibitory effect on ${ }^{35} \mathrm{~S}$-thiamine transport, indicating that the hydroxyethyl group has only a secondary role in the binding of thiamine to the carrier.

f) Effect of sulfur atom. In the previous paper (3), it was shown that pyrithiamine which has the structure substituted by a $-\mathrm{CH}=\mathrm{CH}-$ group for S-Atom of thiazole ring inhibited thiamine active transport competitively. This result suggests that the sulfur atom has no direct role in the interaction between thiamine molecule and the carrier.

g) Effects of pyrimidine and thiazole moieties. The effect of the partial structure of thiamine moiety, such as 2-methyl-4-amino-5-aminomethyl pyrimidine and $\mathrm{N}, 4-$ dimethyl-5-hydroxyethyl thiazole, on the transport of ${ }^{35} \mathrm{~S}$-thiamine was 
examined. As listed in Table 1-g, both pyrimidine and thiazole moieties of thiamine did not inhibit thiamine uptake to any appreciable extent. The quarternary compounds such as dodecylpyridinium chloride and acetylcholine iodide did not show any significant inhibition on ${ }^{35}$ S-thiamine transport.

\section{Inhibition of thiamine transport by thiamine pyrophosphate}

In order to see if thiamine analogues inhibit thiamine uptake from the outside of absorptive cells of the rat small intestine, the effect of thiamine pyrophosphate on the thiamine uptake was examined at the very beginning of the incubation. The time course of the thiamine transport and its inhibition by thiamine pyrophosphate is shown in Fig. 1. The curve for thiamine transport in the presence of thiamine pyrophosphate intersects the origin, indicating that full inhibition is taking place from the very beginning of the incubation. This result rules out the possibility that thiamine pyrophosphate affects thiamine uptake from the inner side of the membrane.

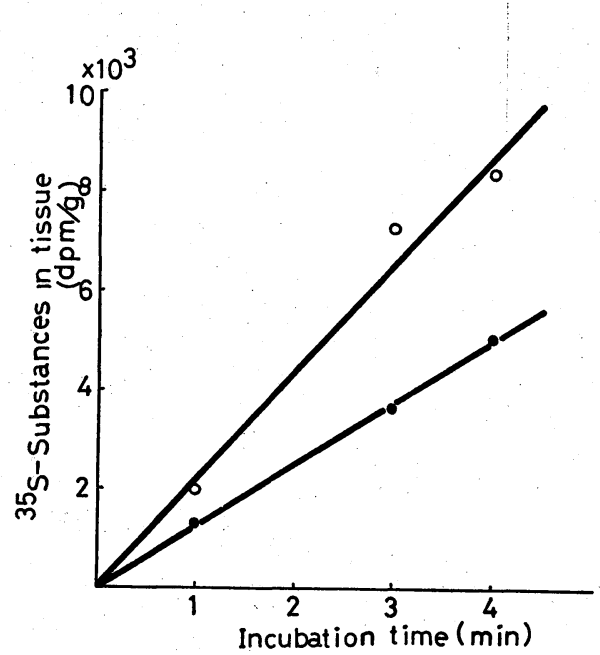

Fig. 1. Inhibition of ${ }^{35}$ S-thiamine uptake by thiamine pyrophosphate. Everted ring segments of the rat small intestine were incubated with $0.6 \times 10^{-6} \mathrm{M}^{35} \mathrm{~S}$-thiamine in the presence (-O) and absence $(\mathrm{O}-\mathrm{O})$ of $6 \times 10^{-6} \mathrm{M}$ thiamine pyrophosphate at $37^{\circ} \mathrm{C}$ for the indicated time periods.

\section{Thiamine transport at various $p H s$}

It is well known that thiamine can be present as three different forms depending upon the $\mathrm{pH}$ of the medium $(4,5)$. Then, in order to obtain further informations on the structural requirement for thiamine transport, thiamine uptake by the rat small intestine was examined at various pHs. The formal charge of thiamine also measured at various $\mathrm{pHs}$ by paper electrophoresis. As shown in Fig. 2, the rate of thiamine uptake was maximum at a $\mathrm{pH}$ range of from 
5.0 to 7.4, wherein thiamine is monocationic as depicted in Fig. 3-A. In the pH range higher than about 9.0, thiamine lacks the quarternary nitrogen and in the $\mathrm{pH}$ range lower than about 4.0, nitrogen atom at 1 '-position of pyrimidine moiety is protonated as depicted in Fig. 3-B and 3-C, respectively. Corresponding to the structural change, the amount of ${ }^{35} \mathrm{~S}$-thiamine taken up by the rat small intestine decreased significantly.

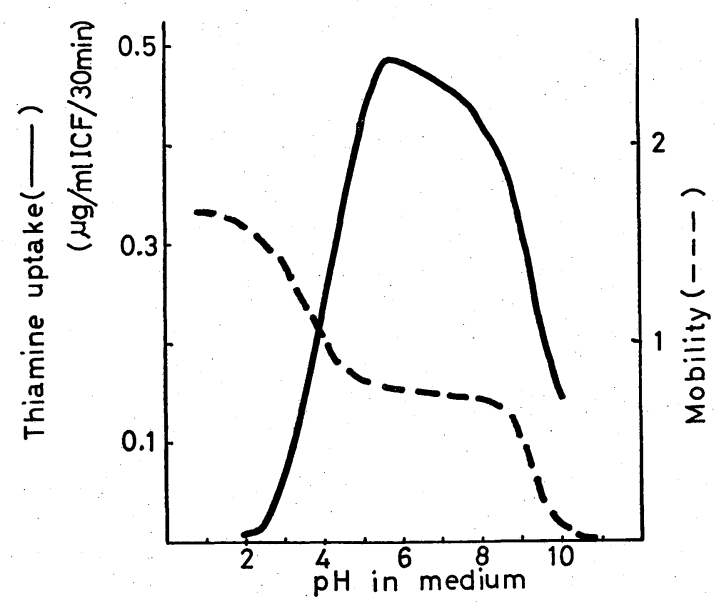

Fig. 2. Effect of $\mathrm{pH}$ on ${ }^{35} \mathrm{~S}$-thiamine uptake by rat intestinal segments and on the formal charge of ${ }^{35}$ S-thiamine. Everted intestinal ring segments were incubated with $0.2 \mu \mathrm{g} / \mathrm{ml}$ ${ }^{35} \mathrm{~S}$-thiamine at various $\mathrm{pH}$ for $30 \mathrm{~min}$ at $37^{\circ} \mathrm{C}$. Formal charge of thiamine was determined by paper electrophoresis.<smiles>Cc1ncc(CN(C=O)C(C)C(S)(CO)CCO)c(N)n1</smiles>

Fig. 3. Chemical structure and formal charge of thiamine in the solution.

Relationship between thiamine transport system and other transport system

The interaction between sugars and amino acids during their active transport in the rat intestine has been known to occur (6). Thus, thiamine uptake was investigated in the presence of sugars or amino acids in the incubation medium. As listed in Table 2, alanine and leucine did not show any inhibitory effect on the thiamine transport. Galactose which is actively transported did not inhibit the thiamine transport at all. While, glucose which is also actively transported stimulated thiamine uptake by the rat small intestine. Fructose, which is ab- 
sorbed by passive diffusion from the rat small intestine, also stimulated thiamine uptake.

Table 2. Effect of sugars and amino acids on thiamine transport. ${ }^{a}$

\begin{tabular}{lcc}
\hline Additive & $\begin{array}{c}\text { Concentration } \\
(\mathrm{mM})\end{array}$ & $\begin{array}{c}\text { Thiamine uptake } \\
(\% \text { to control } \pm \text { S.E. })\end{array}$ \\
\hline L-alanine & 10 & $101.3 \pm 8.8^{\mathrm{b}}$ \\
L-Leucine & 10 & $102.1 \pm 11.2$ \\
D-Galactose & 10 & $100.2 \pm 6.5$ \\
D-Glucose & 10 & $146.8 \pm 6.5$ \\
D-Fructose & 10 & $135.5 \pm 11.6$ \\
\hline
\end{tabular}

a Everted ring segments of the rat small intestine were incubated with $0.6 \times 10^{-6} \mathrm{M}$ ${ }^{35} \mathrm{~S}$-thiamine with or without $10 \mathrm{~mm}$ of sugars or amino acids for $30 \mathrm{~min}$ at $37^{\circ} \mathrm{C}$. Other experimental details are described in EXPERIMENTAL.

${ }^{b}$ Each value represents the average from three experiments.

\section{DISCUSSION}

The results presented in this paper indicate that the amino group and the quarternary nitrogen atom of thiamine molecule are required for the active transport of thiamine from the rat small intestine, and that 2'-methyl and 5-hydroxyethyl groups also influence the transport of thiamine significantly.

In the previous paper (3), it was shown that thiamine is actively transported from the rat small intestine and that thiamine analogues, such as pyrithiamine and chloroethylthiamine, inhibited thiamine transport competitively. In this paper, it was shown, furthermore, that thiamine analogues inhibit thiamine uptake by the rat small intestinal segments from the outside of the cells. These results indicate that thiamine analogues inhibit thiamine absorption from the rat small intestine by competition with thiamine for binding to the carrier. Thus, the decrease or loss of inhibitory effect of non-labeled thiamine to ${ }^{35} \mathrm{~S}$-thiamine transport after modification of a certain part of thiamine structure are considered to mean that the part is required for binding to the binding site of the carrier.

The data in Table 1-a and 1-c indicate that the amino group and the quarternary nitrogen atom are essential for thiamine active transport, respectively. The observation that ${ }^{35} \mathrm{~S}$-thiamine uptake decreased significantly at the $\mathrm{pH}$ range higher than 9.0, wherein the nitrogen is converted from quarternary to tertiary, also indicates the necessity of the quarternary nitrogen. The fact that the modification of either amino group or quarternary nitrogen of thiamine results in almost perfect loss of inhibitory effect on thiamine transport indicates that both of them are required simultaneously for the active transport. The result that the compounds which correspond to the partial structure of thiamine could not depress thiamine transport also supports the above view (Table 1-g).

Thiamine uptake was considerably decreased in the $\mathrm{pH}$ range lower than 
4.0, suggesting that the protonation of nitrogen atom of 1 '-position weakens the affinity of thiamine to the binding site of the carrier. In the coenzyme action of thiamine, it is well known that the 2-position of thiazole moiety is the most important active site, at which a substrate is combined with thiamine pyrophosphate and activated (7). Then, it is interesting that this position seems to play no role in the binding to the carrier.

Non-labeled thiamine showed a higher inhibition to ${ }^{35} \mathrm{~S}$-thiamine uptake than all analogues modified at the 5-position, indicating that the group at this position plays a secondary role for thiamine active transport and that the binding site of the carrier has higher affinity to hydroxyethyl group at this position than other substituents.

These observations on the structural requirement for the intestinal transport of thiamine can be brought together in terms of crude conceptual model as depicted in Fig. 4.

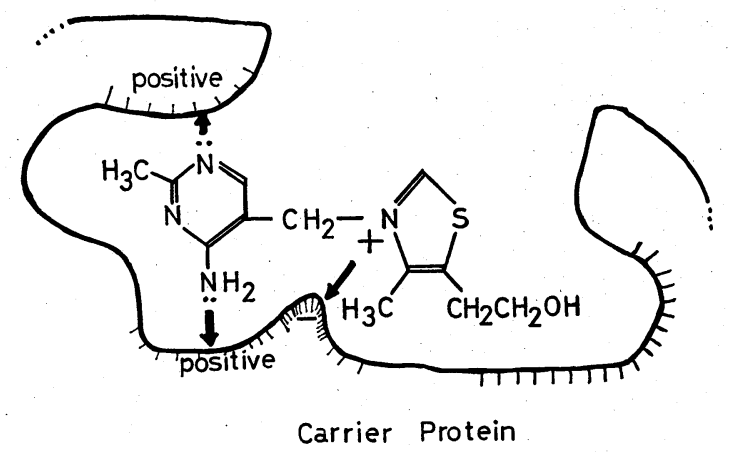

Fig. 4. Conceptual model of the intestinal thiamine transport system.

Recently it was shown that sugars which are actively transported inhibit the active transport of amino acids and vice versa, although the mechanism of the interaction is not clarified. However, sugars and amino acids tested, which are actively transported from the rat small intestine, did not inhibit the thiamine transport. Reversely, metabolizable sugars such as glucose and fructose stimulated thiamine transport and non-metabolizable sugar, galactose, did not. This fact suggests that the stimulation might be caused by the supply of energy for the thiamine transport from the sugar metabolism.

The authors express their deep gratitude to Dr. G. Sunagawa, director of this laboratories, for his valuable advices and encouragements. This work was done in partial satisfaction of the requirements for the Ph.D. degree of T. Komai from Kyoto University.

\section{REFERENCES}

1) Cíane, R. K., Physiol. Rev., 40, 789 (1960). 
2) Spencer, R. P., Am. J. Clin. Nutr., 22, 292 (1969).

3) Komai, T., Kawai, K., and Shindo, H., J. Nutr. Sci. Vitaminol., 20, 163 (1974).

4) Williams, R. R., J. Am. Chem. Soc., 57, 1856 (1935).

5) Mizukami, S. and Hirai, E., J. Org. Chem., 31, 1199 (1966).

6) Cori, C. F., Proc. Soc. Exp. Biol. Med., 24, 125 (1926).

7) Krampitz, L. O., Ann. Rev. Biochem., 38, 213 (1969). 